\title{
Computational modeling of failure in composite structures including uncertainties in material and geometrical properties
}

\author{
Wooseok Ji*, Anthony M. Waas ${ }^{\dagger}$ and Wei Heok $\mathrm{Ng}^{\ddagger}$ \\ University of Michigan, Ann Arbor, MI 48109 \\ Peter A. Gustafson ${ }^{\S}$ \\ Western Michigan University, Kalamazoo, MI 49008 \\ Evan J. Pinedaq \\ University of Michigan, Ann Arbor, MI 48109 \\ Ravi S. T. Raveendrall \\ Comet Technology Corporation, Ann Arbor, MI 48108, USA
}

\begin{abstract}
This paper is concerned with a progressive failure analysis methodology for fiber reinforced composite laminates combining various analytical models designed for investigating failure mechanisms at different length scales. The methodology here employs a fundamental mechanism based approach to predict failure or damage initiation with strong coupling between the multiple length scales. The discrete cohesive zone model elements are used to model the adhesion and delamination failure at macroscale while Schapery theory, a continuum damage theory based on thermodynamics, is used to model material degradation occurring at the lamina level. Furthermore, the present numerical framework is incorporated with a probabilistic analysis module, based on the NEESUS software, to consider material variability and manufacturing inconsistencies. The combined analysis modules are implemented in a non-linear finite element code for modeling the progressive failure of advanced composite structures. The proposed progressive failure analysis methodology is applied to several cases for validating its capability of predicting the evolution of the interactive failure mechanisms in composite structures.
\end{abstract}

\section{Introduction}

The failure of a composite laminate is a result of progressively developing and competing failure mechanisms at multiple length scales. These length scales can be classified as the laminate level, the lamina level, and the microscale fiber/matrix level. Typical failure modes at each length scale are buckling or delamination at the laminate level, matrix microcracking and kink banding at the lamina level, and fiber breaks and fiber/matrix debonding at the fiber/matrix level. When one of these failure modes is initiated, other failure modes may be triggered as the damage due to the initial failure grows. These mixed and interactive failure mechanisms degrade the functional performance of a composite laminate culminating in the collapse of the

\footnotetext{
${ }^{*}$ Research Fellow, Department of Aerospace Engineering

${ }^{\dagger}$ Felix Pawlowski Collegiate Professor, Department of Mechanical and Aeronautical Engineering, Fellow of AIAA.

$\ddagger$ Research Fellow, Department of Aerospace Engineering

$\S$ Professor, Department of Mechanical and Aeronautical Engineering

${ }^{\top}$ Graduate Student Research Assistant, Department of Mechanical Engineering

" President
} 
structure. The present progressive failure analysis (PFA) is targeted at development of a methodology for predicting an initial failure mode and a consequent damage growth coupled with other failure mechanisms at different length scales in a composite structure. To this end, various analytical models are implemented in the PFA presented here, and several examples are chosen to illustrate the modeling that is proposed.

\section{Probabilistic Finite Element Analysis}

The discrete cohesive zone model (DCZM) in Gustafson $(2008)^{1}$ is employed here to analyze the failure of adhesively bonded interfaces and the interlaminar behavior in a composite laminate. The DCZM implements decohesion as a point-wise discrete softening traction law composed of nonlinear 1D elements, connected to node pairs of adjoining surfaces. The DCZM element definition for finite element analysis (FEA) is similar to the conventional continuum elements as shown in Figure 1. Since the DCZM is developed in a discrete sense, it can be easily implemented into the conventional finite element (FE) framework. Incorporation of the DCZM elements into FE models also implies that the various failure modes such as material failure, crack propagation, and local buckling are tracked simultaneously, thus any potential interaction between the failure modes can be captured. The latter is especially important for laminated composites which are known to fail through a combination of failure modes that become active simultaneously or nearly so. The detailed formulation of the DCZM is described in Gustafson $(2008)^{1}$ and De et al. (2005). ${ }^{2}$

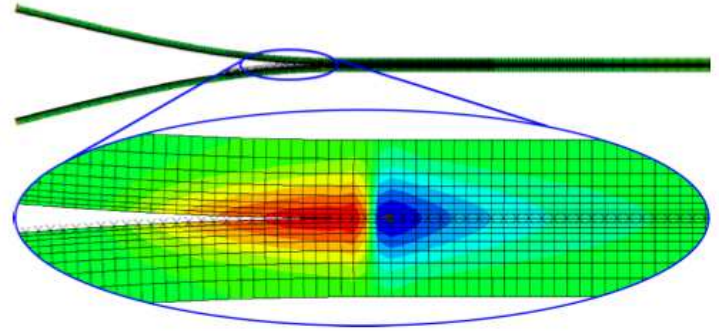

(a)

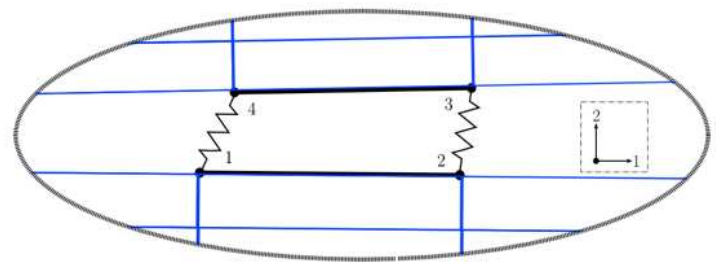

(b)

Figure 1. (a) Typical decohesion process zone (b) Four-node 2D DCZM element with surrounding elements.

The quasi-brittle behavior of epoxy matrix composites is a result of damage accumulation at micro-length scales. In tension, fiber reinforced laminates are affected by the growth of matrix microcracking, distributed throughout the matrix phase of a composite. ${ }^{7}$ Progressive evolution of the microdamage is the primary cause of gradual reduction in the stiffness of the resin until its effects are superseded by larger damage mechanisms, such as transverse cracking. In compression, damage in fiber reinforced laminates manifests via fiber kinking. ${ }^{9}$ Fiber rotation in compression induces large, localized shear strains, which promote further damage in the matrix. This interaction between fiber rotation and matrix damage leads to kinking and finally fracture of the fibers. Although this is a fiber damage mechanism, it is governed by the local tangent shear stiffness of the surrounding matrix.

Schapery developed a thermodynamically based work potential model that is capable of capturing these microdamage mechanisms responsible for the matrix degradation while the fiber direction response was assumed to be linear. ${ }^{5}$ Schapery theory (ST) is extended in Basu et al. $(2006)^{3}$ considering the state of the lamina beyond the first failure in the fiber direction to achieve the successful progressive failure analysis schemes for various loading conditions including compression. Under compressive loading, laminated composite materials are also prone to fail by delaminations due to sublaminate buckling leading to the delamination growth. The discrete cohesive zone model (DCZM) is combined with ST here to model and predict delamination initiation and growth dominated by buckling deformation and the local stiffness degradation by matrix microcracking.

A significant number of modeling parameters are involved in the progressive failure analysis (PFA) at multiple scales with numerous failure mechanisms. Uncertainty in those modeling parameters such as material variability and manufacturing inconsistencies requires a probabilistic approach in PFA. When the uncertainty in the various parameters is considered through a probability-based analysis, the predictive accuracy of the finite element (FE) analysis can be quantified for practical use. The present progressive failure analysis is combined with NESSUS, a modular computer software system for performing probabilistic and uncertainty 
analysis of mechanical components and systems. ${ }^{6}$ This module is implemented in the PFA to compute the probabilistic response of the composite structure. Probabilistic sensitivity studies are also performed using the probabilistic analysis module to identify key material and manufacturing parameters. The stochastic analysis capability with the present PFA methodology provides rational predictions for margins of safety, and quantifying uncertainty.

\section{Applications of the PFA}

\section{III.A. Compressive response of a 2D three-layer composite model}

The present PFA methodology is applied to the two-dimensional (2D) plane strain compression response of a three layer $[90 / 0 / 90]_{1}$ micro laminate geometry as shown in Figure 2. The model has a length of $132 \mu \mathrm{m}$ and width of $64.5 \mu \mathrm{m}$. The fiber volume fraction of the composite is 0.68 and the fiber and matrix materials are assumed to be isotropic and the matrix is assumed to have an elastic-plastic response. In Basu et al., ${ }^{4}$ the modeling of the matrix is done as a damaging Schapery solid and it is found that such a model and a corresponding elastic-plastic model for the matrix leads to the prediction of very similar maximum loads at kink formation.

The mesh in Figure 2 is perturbed using the first buckling mode by a factor of 0.1. This implies that the nodes in the original mesh are modified by a maximum of $0.1 \mu \mathrm{m}$. The discrete cohesive zone model (DCZM) elements are inserted between the interface of $0^{\circ}$ and $90^{\circ}$ plies in order to parametrically study competing failure mechanisms between delamination failure and buckling. The composite is compressed under displacement controlled loading. The analysis is done using ABAQUS/Explicit together

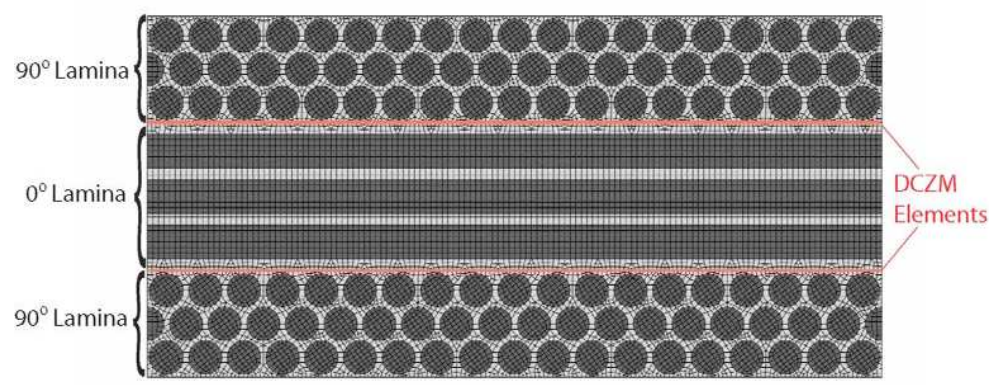

Figure 2. 2D plane-strain model of the 3 layer $[90 / 0 / 90]_{1}$ composite with DCZM elements with NESSUS for performing probabilistic and uncertainty analysis on the compressive behavior of the composite model. The uncertainty is introduced through a probability distribution with two parameters: a mean and a standard deviation. The parameter variability to be studied are the fracture toughness values and the stiffness of the fiber and the matrix. This is done in order to explore how delamination interacts with buckling behavior. Three different sets of interfacial properties are considered. The base DCZM elements has the typical properties of epoxy adhesives, and values of two other sets are varied with a factor of 10 to investigate how changes in the interfacial properties may affect the buckling loads of the laminate. These parameters are summarized in Table 1 , where $\mu$ is a mean and $\sigma$ is a standard deviation of each parameter.

Table 1. Mean, standard deviation of the variable parameters for the $2 \mathrm{D}$ composite model

\begin{tabular}{lcccccc}
\multirow{2}{*}{ Properties } & \multicolumn{2}{c}{ DCZM base } & \multicolumn{2}{c}{ DCZM $\times 10$} & \multicolumn{2}{c}{ DCZM $/ 10$} \\
\cline { 2 - 7 } & $\mu$ & $\sigma$ & $\mu$ & $\sigma$ & $\mu$ & $\sigma$ \\
\hline \hline$G_{I C}\left(\mathrm{~kJ} / \mathrm{m}^{2}\right)$ & 5 & 0.5 & 50 & 5 & 0.5 & 0.05 \\
$G_{I I C}\left(\mathrm{~kJ} / \mathrm{m}^{2}\right)$ & 8.5 & 1.5 & 85 & 15 & 0.85 & 0.15 \\
$\sigma_{C}(\mathrm{MPa})$ & 9 & 0.5 & 90 & 5 & 0.9 & 0.05 \\
$\tau_{C}(\mathrm{MPa})$ & 3.6 & 0.75 & 36 & 7.5 & 0.36 & 0.075 \\
$E_{\text {fiber }}(\mathrm{GPa})$ & 276 & 10 & 276 & 10 & 276 & 10 \\
$E_{\text {matrix }}(\mathrm{GPa})$ & 3.016 & 0.05 & 3.016 & 0.05 & 3.016 & 0.05
\end{tabular}

Figure 3 shows the cumulative probability of peak loads for three different sets of DCZM elements. The range and probability of the peak load for each case are obtained based on the variability of each material parameter as listed in Table 1. The overall peak load decreases substantially as the DCZM properties are decreased. Figure 4 shows the importance level of the parameters considered in this. As expected, the 
fiber stiffness is the most significant factor in the responses of the laminate for all three cases because the fiber carries almost all the axial compressive load. It is interesting to note that the fiber stiffness becomes more significant when the DCZM properties are perturbed from the base values. It is obvious that the fiber stiffness is the main factor to determine the overall response of the laminate with strong interfacial properties $(\mathrm{DCZM} \times 10)$. For the case of low fracture toughness, delamination is prone to initiate and progress before buckling of the laminate. After the delamination, the fibers in the $0^{\circ}$ layer still carry almost all the axial load before the laminate fails at its peak load. Thus, the fiber stiffness is again the key parameter to determine the probability of the peak load for the laminate with lower fracture toughness.

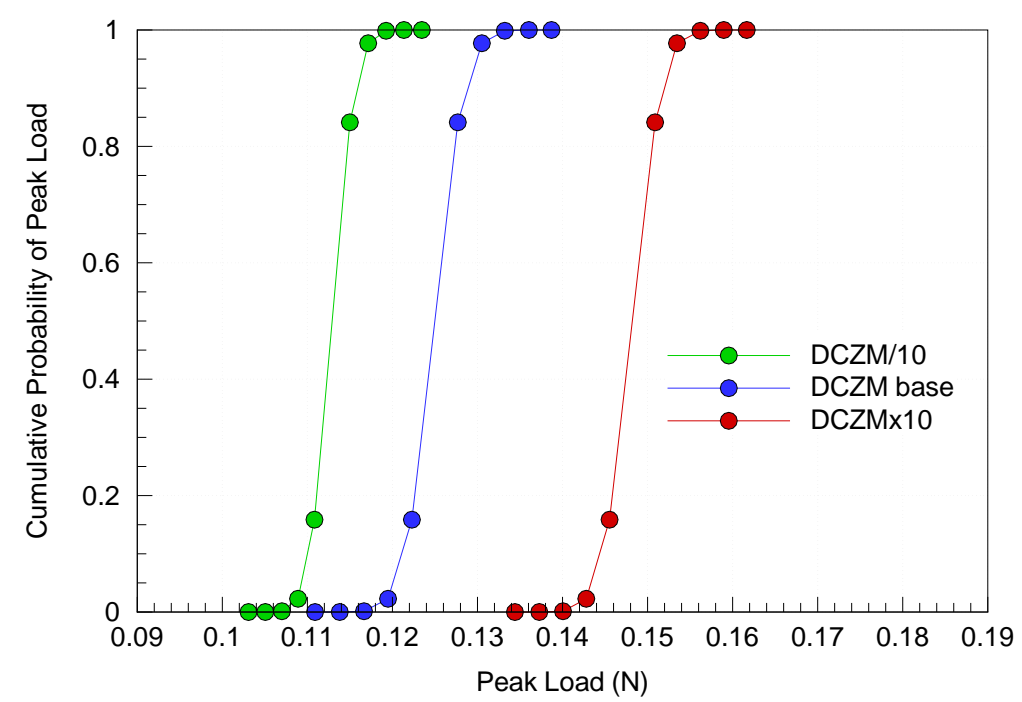

Figure 3. Cumulative probability of peak loads for three different sets of DCZM

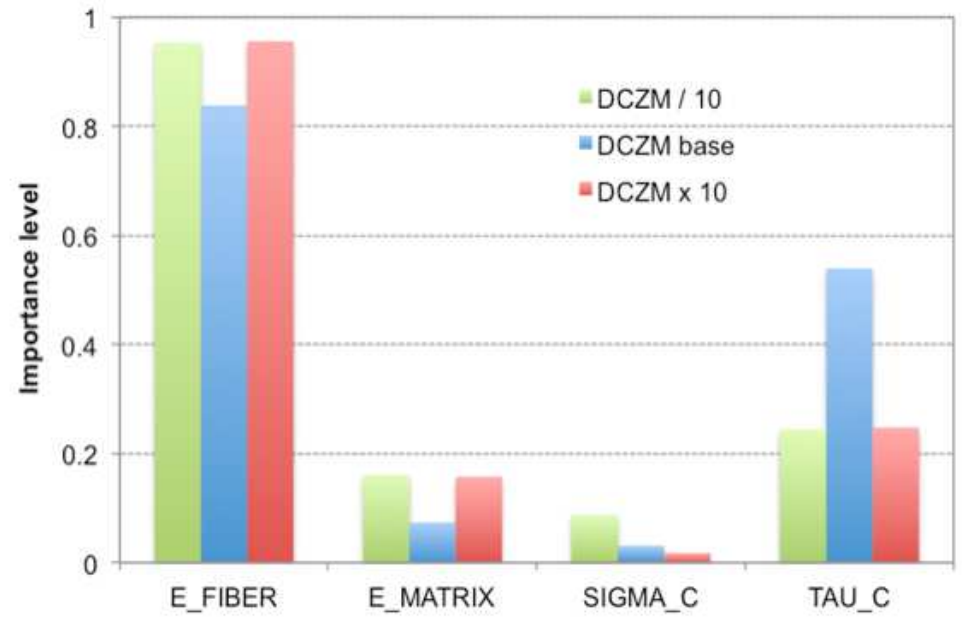

Figure 4. Importance level of modeling parameters

\section{III.B. Assessment of "Pi Joint" Performance under Pull-off Loading}

Adhesively bonded joint technology is now widely used for aircraft structural designs because of its advantage over conventional fastening systems. Adhesively bonded joints can displace stress concentration effects which are unavoidable at fastener areas, and thus lead to improvements in the fatigue resistance of aerospace structures significantly. Furthermore, structural weight can be reduced by replacement of the fastener hardware 
with a corresponding adhesive joint. However, bonded joint design must incorporate failure mechanisms, and corresponding validated analyses methods must be developed to understand mechanical response and performance under a variety of service loads.

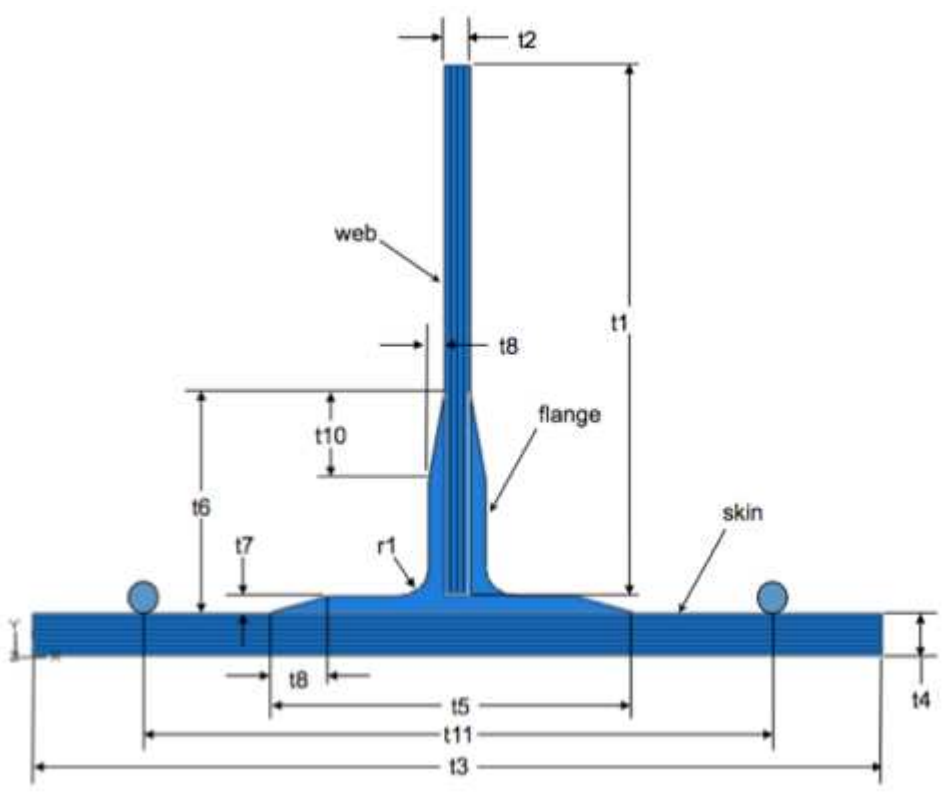

Figure 5. Typical configuration of a Pi-joint composite

Table 2. Dimension of the two-dimensional Pi-Joint configuration. (Unit: $\mathrm{cm}$ )

\begin{tabular}{lcc} 
Part & \multicolumn{2}{l}{ Dimension } \\
\hline \hline \multirow{2}{*}{ Web } & t1 & 3 \\
& t2 & 0.12 \\
\hline Base & t3 & 4 \\
(skin) & t4 & 0.24 \\
\hline \multirow{4}{*}{ Flange } & t5 & 1.75 \\
& t6 & 1.25 \\
& t7 & 0.1 \\
& t8 & 0.075 \\
& t9 & 0.5 \\
& t10 & 0.25 \\
\hline Support & t11 & 3.25
\end{tabular}

In this section, preliminary results from a probabilistic finite element analysis of a typical Pi-Joint are presented, assuming that the joint has no initial disbond at the interfaces. The interfaces are modeled with the DCZM elements to examine the durability of the bonding surface. Representative datasets corresponding to a typical Pi-Joint configuration are used here and Table 2 summarizes the dimensions of the Pi-Joint in Figure 5. The laminate is assumed to have linear elastic material properties. As shown in Figure 5, the $x$-axis is horizontal and the $y$-axis is vertical. Since this is a plane strain analysis (in the $x y$-plane), the properties that are needed for the skin and the web are as follows:

1. For the skin base, the properties are $E_{x}=141 \mathrm{GPa}, E_{y}=6.7 \mathrm{GPa}, G_{x y}=3.2 \mathrm{GPa}$, and $\nu_{x y}=0.33$.

2. For the web, $E_{x}=6.7 \mathrm{GPa}, E_{y}=141 \mathrm{GPa}, G_{x y}=3.2 \mathrm{GPa}$, and $\nu_{x y}=0.33$ are used. 
The through-the-thickness direction of the skin base is aligned with the $y$-axis while that of web is aligned with the $x$-axis. The flange is assumed to have the same material properties as the laminate. The material properties are taken from Rudraraju (2010), ${ }^{11}$ as being a typical lamina material dataset.

Table 3. Mean, standard deviation of the variable parameters

\begin{tabular}{lcccccc}
\multirow{2}{*}{ Properties } & \multicolumn{2}{c}{ DCZM base } & \multicolumn{2}{c}{ DCZM $\times 2$} & \multicolumn{2}{c}{ DCZM $/ 2$} \\
\cline { 2 - 7 } & $\mu$ & $\sigma$ & $\mu$ & $\sigma$ & $\mu$ & $\sigma$ \\
\hline \hline$G_{I C}\left(\mathrm{~kJ} / \mathrm{m}^{2}\right)$ & 2.5 & 0.25 & 5 & 0.5 & 1.25 & 0.125 \\
$G_{I I C}\left(\mathrm{~kJ} / \mathrm{m}^{2}\right)$ & 4.25 & 0.75 & 8.5 & 1.5 & 2.125 & 0.375 \\
$\sigma_{C}(\mathrm{MPa})$ & 4.5 & 0.25 & 9 & 0.5 & 2.25 & 0.125 \\
$\tau_{C}(\mathrm{MPa})$ & 1.8 & 0.375 & 3.6 & 0.75 & 0.9 & 0.1875 \\
$E_{x}(\mathrm{GPa})$ & 141 & 5 & 141 & 5 & 141 & 5 \\
$E_{y}(\mathrm{GPa})$ & 6.7 & 0.05 & 6.7 & 0.05 & 6.7 & 0.05
\end{tabular}

The Pi-Joint is modeled with two-dimensional (2D) plane strain elements, using the commercial code ABAQUS. The model is fixed at the two constraint points and pulled off at the top surface of the web. The DCZM elements are implemented at the interface between the base and the flange, and also the interface between the flange and the web in order to parametrically study delamination failure. The fracture toughness values are listed in Table 8. The analysis is done using ABAQUS/Implicit together with NESSUS, a modular computer software system for performing probabilistic and uncertainty analysis of structural/mechanical components and systems.

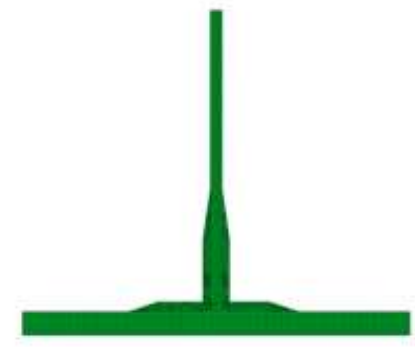

(a)

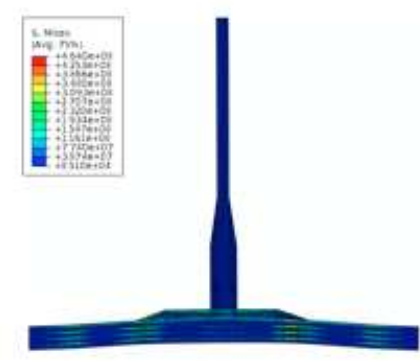

(b)

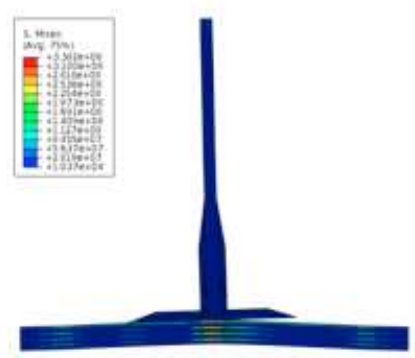

(c)

Figure 6. Progressive failure of the Pi-Joint composite subjected to pulloff load at the top. (a) initial configuration (b) deformed shape at peak load (c) delamination evolution

Figure 6 shows the typical failure of the Pi-Joint where the delamination at the interface between the skin base and flange is observed. Again, the DCZM elements are embedded at the interface between the base and flange as well as the interface between the web and flange. Several modeling parameters have significant influence on the progressive failure of the Pi-joint structure. Of interest are uncertainties of the modeling parameters such as material variability, which require probabilistic approaches in traditional FEA. When the uncertainties in the various parameters are considered through probability-based analysis, the deterministic FE analysis can be quantified for practical use. Additionally, the main parameters driving the dominant failure mechanisms can also be identified and the results can be utilized at a preliminary design stage to improve the model design.

The uncertainty in the material variability is represented as a probability distribution with a mean and a standard deviation. NESSUS coupled with ABAQUS is used to obtain the probability of responses, the random parameter importance levels, and the sensitivities of the probability of responses, based on the mean and the standard deviation values of each parameter. The variable parameters to be studied in this analysis are the fracture toughness values and the stiffness values of the laminate. The material property variability considered in this report is listed in Table 3, where $\mu$ is a mean and $\sigma$ is a standard deviation. Three cases of the DCZM properties are considered by varying the values of the base DCZM elements with a factor of two to investigate the effect of the interfacial properties on the performance of the Pi Joint. Figure 7 shows the cumulative probability of peak loads for the three cases with the variability in material properties and 
cohesive interfaces. Figure 8 shows the importance level of the parameters considered in this probabilistic finite element analysis (PFEA) study.

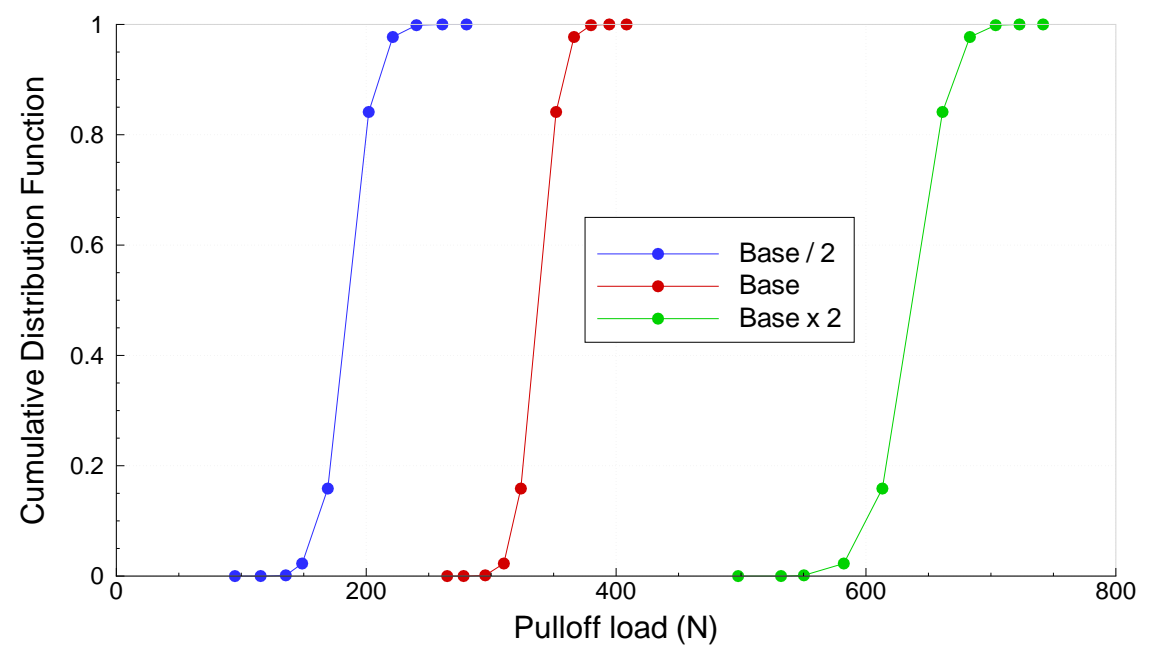

Figure 7. Cumulative probability of pulloff loads at failure from the PFA of Pi-Joint pulloff test

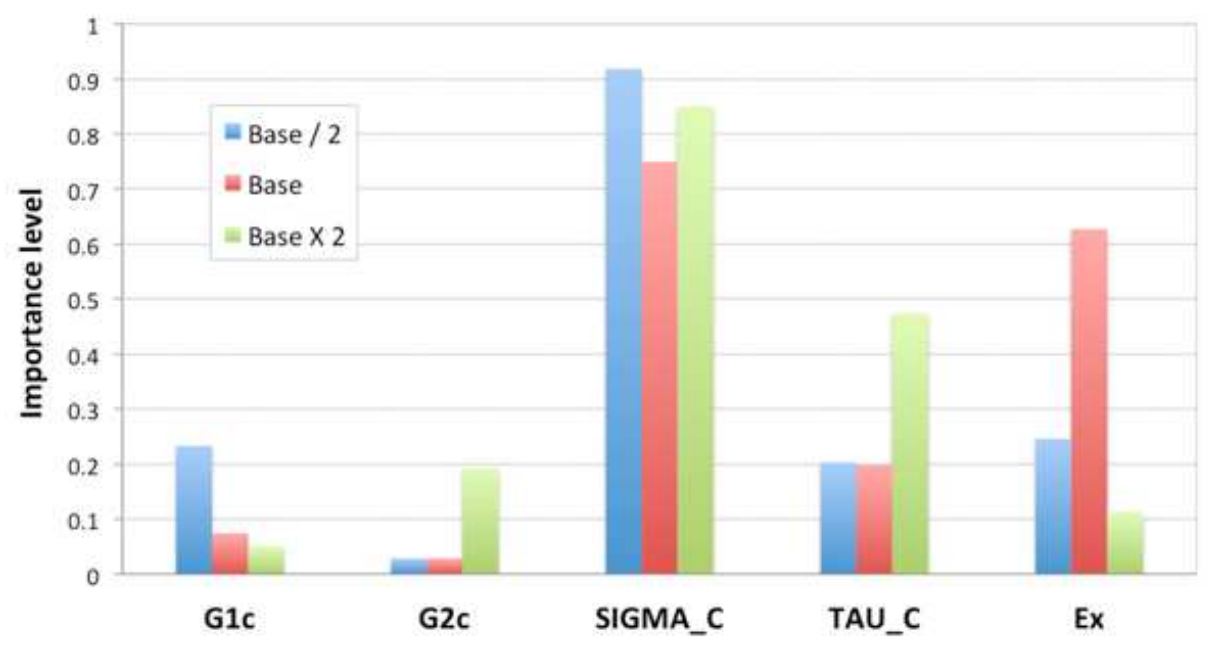

Figure 8. Importance level of modeling parameters

As shown in Figure 7, the fracture toughness values significantly change the peak loads between the three cases. Using each curve in Figure 7, reliability of the Pi Joint sustainability can be evaluated when the modeling variability in Table 3 is considered. Figure 8 displays the importance levels of the given variability parameters that dominates the probability of the failure load. The cohesive tensile strength and the lamina modulus in $x$-direction appear to be the main factors defining the performance of the Pi Joint. From the failure mode in Figure 6, the Pi Joint is susceptible to failure in the interface between the flange and the base. High tension is developed in the interface region and, thus, the cohesive strength, $\sigma_{C}$, of the adhesive layer has a dominant influence on the performance of the Pi Joint considered here.

\section{III.C. Progressive Damage Growth of a Composite Plate with an Initial Delamination}

The present PFA scheme, ST coupled with DCZM, is applied to study progressive delamination growth in a flat plate subjected to axial compression loading as illustrated in Figure 3 of. ${ }^{12}$ A flat 9.0 in. $\times 4.5$ in. composite panel with a centrally located 2.5-inch-diameter delamination is compressed along its long axis. 
The laminate stacking sequence of the panel is $\left[(\mp 45 / 90 / 0)_{2} / \mp 60 / \mp 15\right]_{S}$. The delamination is placed at the interface between the 5th and 6th ply (between $-45^{\circ}$ and $45^{\circ}$ ). The panel is made of an AS4/3501-6 graphite/epoxy composite material system for which the lamina properties are available in Sicking (1992). ${ }^{10}$ The fracture toughness values for Modes I, II, and III at the delamination interface are also listed in Table 4.

Table 4. Modeling input for the fracture toughness values of AS4/3501-6 graphite/epoxy material.

\begin{tabular}{lc} 
Property & Value \\
\hline \hline$G_{I C}(\mathrm{lb} / \mathrm{in})$ & 0.46863 \\
$G_{I I C}(\mathrm{lb} / \mathrm{in})$ & 3.171825 \\
$G_{I I C}(\mathrm{lb} / \mathrm{in})$ & 3.171825 \\
$\sigma_{I C}(\mathrm{psi})$ & 20 \\
$\sigma_{I I C}(\mathrm{psi})$ & 120 \\
$\sigma_{I I C}(\mathrm{psi})$ & 120
\end{tabular}

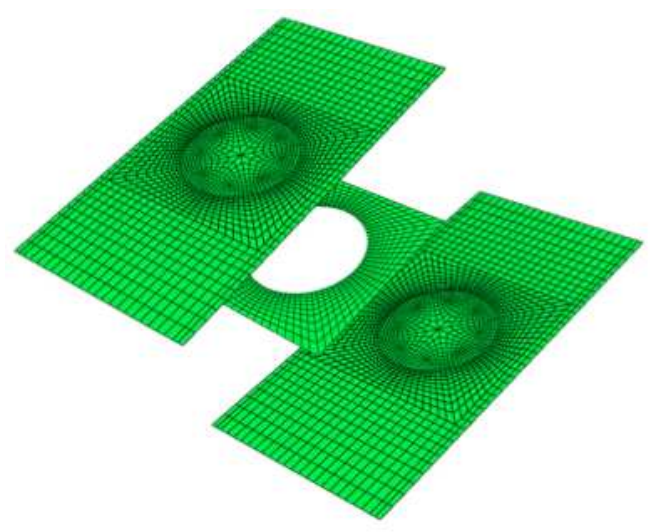

Figure 9. FE modeling of the composite panel with the initial delamination utilizing the DCZM elements

The composite panel is modeled as three parts; an upper sub-laminate, a zero thickness DCZM layer, and a lower sub-laminate, as shown in Figure 9. The upper sub-laminate represents the first five layers and the lower sub-laminate corresponds to the rest of the lamina stack. The material behavior is assumed to be elastic orthotropic. As will be shown later, the transverse and shear moduli of the adjacent layers to the initial delamination plane degrade as microdamage grows in the matrix phase. Progressive damage is modeled using Schapery theory, which is implemented into the PFA framework. The DCZM layer is designated for modeling the initial delamination at the interface between the 5 th and 6 th layers, and also for predicting the delamination growth around the region.

Figure 10 shows the section views of the composite plate at different locations along the width. The delamination pattern agrees well with the experimental observations reported by Reeder et al. (See Figure 10 of Reeder et al. ${ }^{12}$ ). Figure 11 shows the distribution of the degraded shear stiffness at the upper and lower interface. Since the upper sub-laminate has lower compressive strength than the lower sublaminate because of the fewer number of layers, the upper sub-laminate experiences more severe deformation during the compression. As a result, the shear stiffness degradation progresses significantly near the circular delamination region, leading to the differences between the upper and lower sub-laminates as shown in Figure 11. In the lower sub-laminate, the degradation of the shear stiffness is not as obvious as that of the upper sub-laminate. The mismatched region of the degraded shear stiffness coincides with the delamination pattern shown in Figure 12.

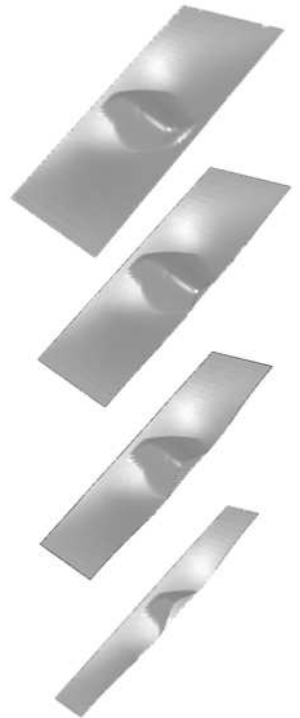

Figure 10. Section views of delamination pattern 

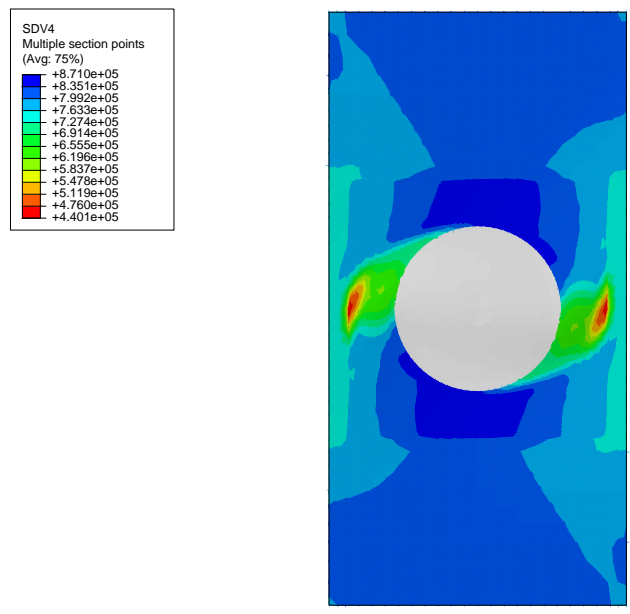

(a)
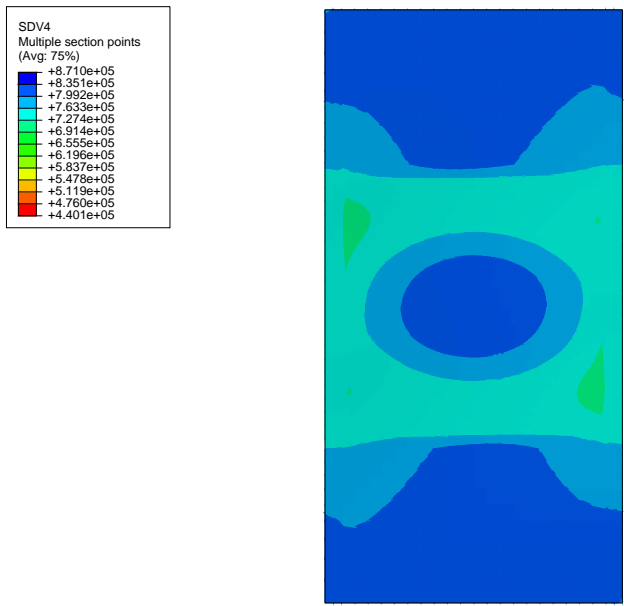

(b)

Figure 11. Distribution of degraded shear modulus (a) at 5th layer and (b) at 6th layer

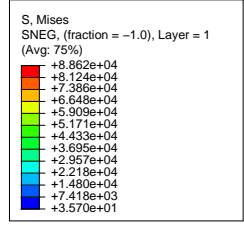

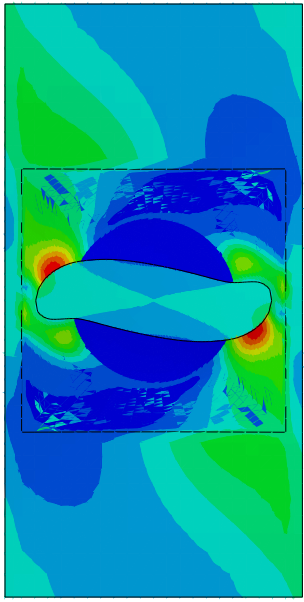

Figure 12. Delamination pattern with Von Mises stress distribution 


\section{Conclusions}

A reliability based progressive failure analysis methodology is developed here by implementing a matrix microcracking damage model based on Schapery theory coupled with the discrete cohesive zone model for interfacial failure. The numerical PFA framework employs a mechanistic, failure mechanism based approach that is devoid of empirical formulas that have dominated failure prediction tools in composite laminate failure prediction technologies. Probabilistic approaches are incorporated with the PFA to account for material variability and manufacturing inconsistencies, and to provide reliability based failure indicators. The method presented here is demonstrates that it is capable of predicting the interaction between out-of-plane failure and in-plane failure, by considering the compression response of a simple 3-ply cross-ply composite laminate subjected to compression. The present PFA method has also been shown to be suitable for other applications of progressive failure analysis for complex structural configurations such as "Pi Joints". The prediction from the present PFA provides the necessary guidance in selecting the most robust methodology to achieve the desired goal of combining initial design with reliability based tools to redesign and evaluate existing designs of composite structures.

\section{References}

\footnotetext{
${ }^{1}$ Gustafson, Peter A. and Waas, Anthony M., "Efficient and robust traction laws for the modeling of adhesively bonded joints," 50th AIAA Structural Dynamics and Materials Conference, Schaumburg, IL, 2009.

${ }^{2}$ De, X., Salvi, A.G., Ce, S., Waas, A.M., and Caliskan, A., "Discrete cohesive zone model to simulate static fracture in $2 \mathrm{D}$ triaxially braided carbon fiber composites," Journal of Composite Materials Vol. 40, No. 22, 2005, pp. 2025-46.

${ }^{3}$ Basu, S., Waas, A.M., and D.R. Ambur, "Progressive failure modeling of laminates incorporating material and geometric nonlinearities," 47th AIAA Structural Dynamics and Materials Conference, Newport, Rhode Island, 2006.

${ }^{4}$ Basu, S., Waas, A.M., and D.R. Ambur, "Compressive failure of fiber composites under multi-axial loading," Journal of the Mechanics and Physics of Solids, Vol. 54, 2006, pp. 611-634.

${ }^{5}$ Schapery, R. A., "Theory of mechanical behavior of elastic media with growing damage and other changes in structure," Journal of the Mechanics and Physics of Solids, Vol. 38, No. 2, 1990, pp. 215-215.

${ }^{6}$ Thacker, B.H, Riha, D.S., Fitch S.K., Huyse L.J., and Pleming J.B., "Probabilistic engineering analysis using the NESSUS software," Structural Safety, Vol. 28, No. 1-2, 2006 pp. 83-107.

${ }^{7}$ Schapery R.A. and Sicking D.L., "On nonlinear constitutive equations for elastic and viscoelastic composites with growing Damage," Mechanical Behavior of Materials, Vol. 47, 1995, pp. 45-76.

${ }^{8}$ Schapery R.A., "Prediction of compressive strength and kink bands in composites using a work potential," International Journal of Solids and Structures, Vol. 32, No. 6, 1995, pp. 739-765.

${ }^{9}$ Yerramalli C.S and Waas A.M., "A failure criterion for fiber rein- forced polymer composites under combined compressiontorsion loading," International Journal of Solids and Structures, Vol. 40, No.5, 2003, pp. 1139-1164.

${ }^{10}$ Sicking, D.L., "Mechanical characterization of nonlinear laminated composites with transverse crack growth," Ph.D. Dissertation, Texas A\&M University.

11, Rudraraju, S. S., A. Salvi, Garikipati, K., and Waas, A. M., "In-plane fracture of laminated fiber reinforced composites with varying fracture resistance: Experimental observations and numerical crack propagation simulations," International Journal of Solids and Structures Vol. 47 No. 7-8, 2010, pp. 901-911.

${ }^{12}$ Reeder, J.R., Song, K., Chunchu, P.B., and Ambur, D.R., "Postbuckling and growth of delaminations in composite plates subjected to axial compression," 43rd AIAA Conference, Denver, CO, 2002.
} 